\title{
Low-Timing-Jitter Near-Infrared Single-Photon-Sensitive 16-Channel Intensified-Photodiode Detector
}

\author{
Michael A. Krainak, Wei Lu, Guangning Yang, Xiaoli Sun \\ NASA Goddard Space Flight Center Greenbelt, MD 20771 \\ Derek Sykora, Mike Jurkovic, Verle Aebi, Ken Costello, Richard Burns \\ Intevac, Inc. 3560 Bassett Street, Santa Clara, CA 95054
}

\begin{abstract}
We developed a 16-channel InGaAsP photocathode intensified-photodiode (IPD) detector with 78 ps (1-sigma) timing-jitter, $<500$ ps FWHM impulse response, $>15 \%$ quantum efficiency at $1064 \mathrm{~nm}$ wavelength with $131 \mathrm{kcps}$ dark counts at $15 \mathrm{C}$.

(C2010 Optical Society of America

OCIS codes: (040.5250) Photomultipliers (040.5570) Quantum detectors (280.3420) Laser sensors.
\end{abstract}

\section{Introduction}

Laser-based instruments require single-photon-sensitive detectors to minimize resource requirements (size, weight, power and cost). This is particularly critical for space-based science instruments and optical communications terminals, but is also important for many airborne and ground-based systems. A detector with low timing jitter $(<100 \mathrm{ps})$ is vital for high-precision micropulse lidar topographic mapping systems [1] and high-data-rate (short time slot-width) pulse-position-modulation (PPM) free-space optical communication systems [2]. The most costeffective practical high-peak power pulsed laser systems (e.g. $\mathrm{Nd}, \mathrm{Yb}$ and $\mathrm{Er}$ ) operate at near-infrared wavelengths where space-qualified single-photon detectors have not been available.

The intensified photodiode (IPD) detector, (a.k.a hybrid photomultiplier tube (HPMT) or hybrid photodetector (HPD)), provides high-gain with low-noise suitable for single-photon sensitivity via electron bombardment of an avalanche diode. Advantages of the IPD include large detector area $(1 \mathrm{~mm}$ for a single channel device), high maximum count rates ( $>200 \mathrm{Mcps}$ ), high bandwidth (GHz), low afterpulsing, and near room temperature operation. Further features include low dark noise, large dynamic range for full analog pulse received waveform preservation and photon number resolution. Operating principles and performance results of previous IPDs have been reported for use at visible $[3,5]$, and near-infrared $[4,5,6]$, wavelengths.

\section{Achieving low timing jitter}

In this work, we produced a 16-channel IPD with an InGaAsP photocathode for use at $1064 \mathrm{~nm}$ wavelength. Each channel has a $159 \mu \mathrm{m} \times 159 \mu \mathrm{m}$ sensitive detection area at the photocathode. The overall IPD timing jitter is dominated by the random walk of the minority carrier electron across the InGaAsP absorber layer inside the transferred-electron photocathode. The contribution of this photocathode timing jitter, $\sigma_{j}$, is given [2], by:

$\sigma_{j}=\frac{W^{2}}{2.62 D_{n}}$ where $W$ is the InGaAsP absorber thickness and $D_{n}$ is the electron diffusion coefficient. A

$2.5 \mu \mathrm{m}$ lattice matched InGaAsP absorber layer with a mobility of $3000 \mathrm{~cm}^{2} / \mathrm{V}-\mathrm{s}$, gives a photocathode timing jitter of $307 \mathrm{ps}$. A single-channel device [4] with this geometry had an overall timing jitter of $\sim 500 \mathrm{ps}$ with $26 \%$ quantum efficiency (QE). A four-channel IPD [2] with similar timing jitter was previously demonstrated. For our 16-channel device, we reduced the InGaAsP layer thickness to $0.8 \mu \mathrm{m}$ that provides a measured QE of $15 \%$ at $1064 \mathrm{~nm}$ wavelength. Figure 1 shows the timing jitter (188 ps FWHM, 78 ps one-sigma) measurement results for our $0.8 \mu \mathrm{m}$ thick photocathode. We measured the timing jitter using two independent instruments 1) the Picoquant HydraHarp 400 multichannel scaler providing 188 ps FWHM measurement and an Agilent Model DSA91304A (13GHz BW) oscilloscope that calculated the one sigma jitter directly from the waveform histogram as $78 \mathrm{ps}$. This is an upper limit on the timing jitter because it includes the photon timing uncertainty associated with the $100 \mathrm{ps}$ pulse width of the experimental test laser. For further context, a visible singlechannel HPD with a $1 \mathrm{~mm}$ diameter photocathode has 28 ps timing jitter with $46 \%$ QE at $500 \mathrm{~nm}$ wavelength [3]. 


\section{Additional 16-channel IPD measured characteristics}

The single photon impulse response has a pulse width of $550 \mathrm{ps.} \mathrm{The} \mathrm{adjacent} \mathrm{pixel} \mathrm{cross} \mathrm{talk} \mathrm{was} \mathrm{than} 1.1 \%$ for any pair of adjacent pixels. The internal gain is $>10^{4}$ for each pixel. Figure 2 shows the measured reduction of dark counts per channel with decreasing temperature and the extrapolation prediction of less than $10 \mathrm{kcps}$ at $-20 \mathrm{C}$.

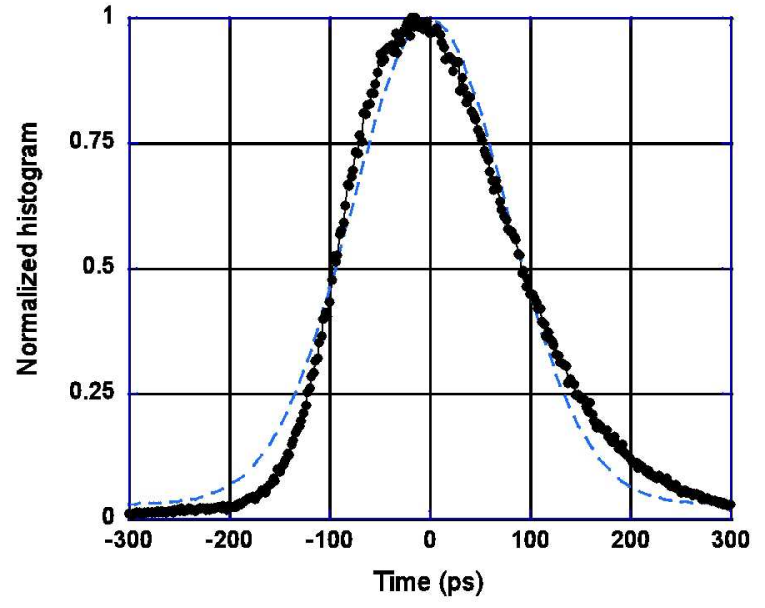

Fig. 1. Single-photon timing jitter measurement results for IPD with $0.8 \mu \mathrm{m}$ InGaAsP layer thickness. $\quad F W H M=188$ ps. Dashed line is Gaussian fit with $\sigma=78$ ps.

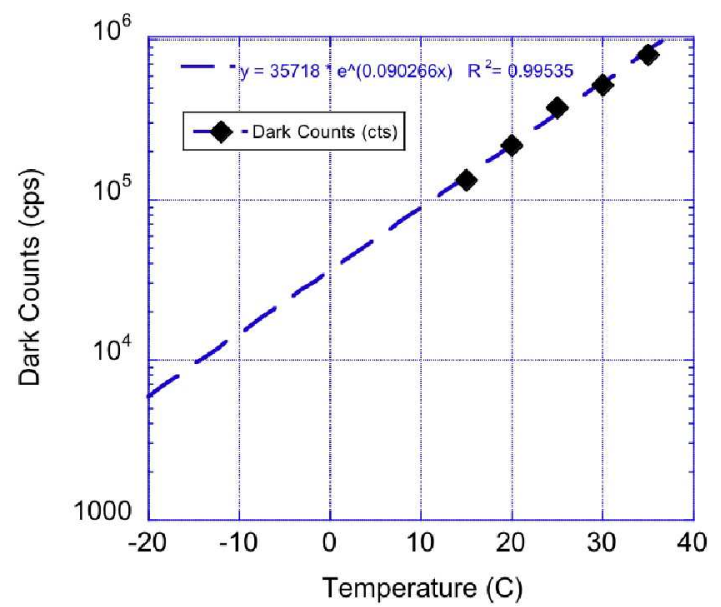

Fig. 2. IPD dark counts per channel vs. Temperature. Experiment $(\diamond)$ and extrapolation (dashed line).

An excess noise factor of 1.2 was measured from the pulse height amplitude distribution. Figure 3 shows excellent agreement for two independent sets of measured photon number resolution (scaled pulse height distribution histogram) and the Poisson probability mass function $f(k, \lambda)=\frac{\lambda^{k} e^{-\lambda}}{k !}$ theory with a) $\lambda=1.6$ and b) $\lambda=3.3$.

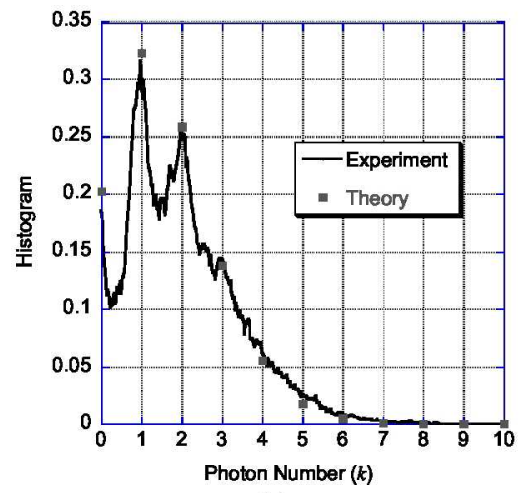

(a)

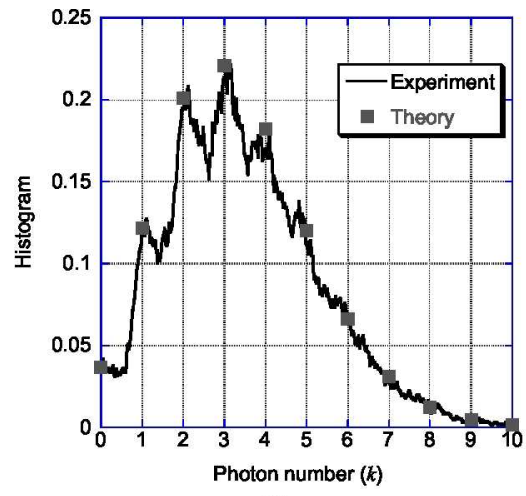

(b)

Fig. 3 Measured scaled histogram of the pulse height distribution and Poisson theory for (a) $\lambda=1.6$ and $b) \lambda=3.3$.

\section{References}

[1] D. McLennan "Ice, Clouds and Land Elevation (ICESat-2) Mission," Proc. SPIE, 7826, 782610 (2010)

[2] A. Biswas, B. Moision, W. T. Roberts, et al. "Palomar receive terminal (PRT) for the Mars laser communication demonstration (MLCD) project," Proceedings of the IEEE 95, 2045-2058 (2007).

[3] A. Fukasawa, J. Haba, A. Kageyama, H. Nakazawa, and M. Suyama "High Speed HPD for Photon Counting," IEEE Transactions On Nuclear Science 55, 758-762 (2008).

[4] X. Sun, M. A. Krainak, W. E. Hasselbrack, W. E., D. F. Sykora, R. La Rue, "Single photon counting at 950 to 1300 nm using InGaAsP photocathode - GaAs avalanche photodiode hybrid photomultiplier tubes," Journal of Modern Optics, 56, 284-295, (2009).

[5] R. A. La Rue, K. A. Costello, G. A. Davis,J. P. Edgecumbe, V. W. Aebi, "Photon Counting III-V Hybrid Photomultipliers Using Transmission Mode Photocathodes," IEEE Trans. Electron Devices 44, 672-678 (1997).

[6] R. A. La Rue, G. A. Davis, D. Pudvay, K. A. Costello, V. W. Aebi, "Photon Counting 1060-nm Hybrid Photomultiplier with High Quantum Efficiency," IEEE Electron Device Lett. 20, 126-128 (1999). 